\title{
A NOTE ON CHARACTERIZATION OF MOISHEZON SPACES
}

\author{
K. RAMA
}

\begin{abstract}
In this nole a necessary and sufficient condition for a compact complex space $Y$ to he Moishezon is obtained; it can be seer as the existence of a line bundle $L$ on $X$ such that, for some point $x \in X$, the first cohomology groups of $X$ with values respectively in $L \otimes m_{x}$ and $L \otimes m_{x}^{2}$, vanish. (Here $m_{x}$ denote the ideal sheaf at $x$ ).
\end{abstract}

1. Grauert and Riemenschneider [1], [7] conjectured a characterization of a Moishezon space in terms of "almost (quasi) positive" coherent sheafs on it. The problem in proving this was to obtain Moishezonness of a compact complex space $X$ if it carries an almost positive coherent sheaf. From then a number of characterizations of Moishezonness with additional assumptions to the hypothesis of the conjecture have been obtained [7], [12], [9], [5], [10]. For example, in [8] it was assumed that $X$ is Kähler. Some of these are in such a way that the proofs can be obtained by using Kodaira's techniques: namely blowups, Kodaira's Vanishing and embedding theorems. Siu [11] has succeeded in proving a stronger version than the conjecture. His proof is by using the powerful theorem of Hirzebruch-Riemann-Roch and giving estimates on the dimensions of the cohomology groups of $X$ with coefficients in a power of a line bundle which has a non strictly positive curvature form. It deals, more generally with one of the fundamental questions of obtaining holomorphic sections for non strictly positive line bundles.

The present note gives a characterization of Moishezon spaces using Kodaira's techniques.

2. Since compact complex analytic space can be desingularized and coherent analytic sheaves can be made free (modulo torsion) by proper modifications, a characterization of Moishezon space $X$ can be stated in terms of compact complex manifold $X$ and line bundles (locally free sheaves) over $X$. We prove the following: 
Theorem. Let $X$ be an irreducible, $n$-dimensional compact complex manifold. Then $X$ is Moishezon (i.e. the transcendence degree of the field of meromorphic functions on $X$ is equal to the complex dimension of $X$ ) if and only if there exists a line bundle $L$ over $X$ such that for $r=1,2$,

$$
H^{1}\left(X, L \otimes m_{x}^{r}\right)=0 \text { for some point } x \in X
$$

( $m_{x}$ denotes the ideal sheaf of $x$ ).

Proof: To prove the "if" part, consider the exact sequences

$$
0 \longrightarrow m_{x}^{r} \longrightarrow \mathcal{O}_{X} \longrightarrow \mathcal{O}_{X} / m_{x}^{r} \longrightarrow 0, r=1,2
$$

Then one can deduce the exactness of

$$
H^{0}(X, L) \stackrel{{ }^{a} r}{\longrightarrow} H^{0}\left(X, L \otimes \mathcal{O}_{X} / m_{x}^{r}\right) \longrightarrow H^{1}\left(X, L \otimes m_{r}^{r}\right) \text { for } r=1,2
$$

using the given vanishing for $r=1$, we get from (a) that the map $\alpha_{r}$ is onto. This means that the global holomorphic sections of $L$ generate the stalk $L_{2}=$ $H^{\circ}\left(X, L \otimes \mathcal{O}_{X} / m_{x}\right)$. Hence the meromorphic mapping $s: X \rightarrow \mathbb{P}^{m}$ induced by a basis $s_{1}, \ldots, s_{m}$ of $H^{0}(X, L)$ is holomorphic at $x \in X$ and separates points in a neighbourhood of $x$.

For $r=2$, the sequence (a) gives the surjection of $\alpha_{2}: H^{0}(X, L) \rightarrow H^{0}(X, L \otimes$ $\mathcal{O}_{X / m_{x}^{2}}$. This implies that $s$ has maximal rank at $x$. That is, $s$ defines a closed embedding near $x$. Then for a suitable neighbourhood $U$ of $x$ in $X$, there exist meromorphic functions $f_{1}, \ldots, f_{n}$ on $\mathbb{P}^{m}$ such that $d\left(f_{\left.1\right|_{a\{U\}}}\right) \wedge \cdots \wedge$ $d\left(f_{\left.n\right|_{\text {o(I) }}}\right)(s(x)) \neq 0$ (since $s(U)$ becomes on $n$-dimensional complex submanifold of an open subset of $P^{m}$ ). Since $s$ is meromorphic, the functions $f_{1}, \ldots, f_{n}$ can be lifted to meromorphic functions $g_{1}, \ldots, g_{n}$ on $X$ which are algebraically and analytically independent with $d g_{1} \wedge \cdots \wedge d g_{n}(x) \neq 0$ (by a theorem in [6]). Thus $X$ is Moishezon. Conversely assume that $X$ is Moishezon. Then by Theorem 4 of [7] there exists a line bundle $H$ on $X$ which is positive on a dense open set $U$. Let $x \in U$. Consider the blow up $(\tilde{X}, \Pi)$ of $X$ at $x$. Let $E_{x}$ be the line bundle on $\tilde{X}$ associated to the divisor $\Pi^{-1}(x)$. Then $E_{\left.x\right|_{n-1} ^{*}(x)}$ is positive by standard arguments [2], [3], [7]. Since $\Pi^{*} H$ is semipositive everywhere and positive on $\Pi^{-1}(U-x)$ there exists a positive integer $\mu$ such that for $r=0,1, T=\Pi^{*} H^{\nu} \otimes E_{x}^{*^{n++}}$ is semipositive everywhere and positive in $\Pi^{-1}(U)$ for all $\nu \geq \mu$ (note that $E^{*^{n+r}}$ is positive in a neighbourhood of $\Pi^{-1}(x)$ and trivial outside $\Pi^{-1}(U)([3])$. Observe that $\tilde{X}$ is Moishezon, being a modification of a Moishezon space [4]. Hence by applying Theorem 3 of [6] for a trivial bundle and $T$, it follows that

$$
H^{l}\left(\tilde{X}, \Pi^{*} H^{\nu} \otimes E_{x}^{*^{n+r}} \otimes K_{\tilde{X}}\right)=0, l \geq 1, \nu \geq \mu, r=0,1 .
$$

By Kodaira's formula [3], $K_{\bar{X}}=\Pi^{*} K_{X} \otimes E_{z}^{n-1}$, we get, in particular,

$$
H^{1}\left(\tilde{X}, \Pi^{*}\left(H^{\mu} \otimes K_{X}\right) \otimes E_{x}^{* r}\right)=0, r=1,2
$$

i.e. $H^{1}\left(X, L \otimes m_{x}^{r}\right)=0, r=1,2$, by taking $L=H^{\mu} \otimes K X$. 


\section{References}

1. H. GRAUERT, O. RIEMENSCHNEIDER, Verschwindungssütze für analytishe kohomologie-gruppen auf komplexen räumen, Invent. Math. 11 (1970), 263-292.

2. Pil. Grif FITHS, Hermitian differential geometry, chern classes and positive vector bundles, Global Analysis, papers in honor of K. Kodaira, Tokyo and Princeton (1969), 185-251.

3. K. Kodalra, On Kähler varieties of restricted type, Ann. of Math. 60, 2 (1954), 28-48.

4. B.G. MolšezoN, On $n$-dimensional compact varieties with $n$ algebraically independent meromorphic functions, Amer. Math. Soc. Translations 63 (1967), 51-177.

5. K. Rama, Ph.D Thesis, University of Hyderabad, INDIA, 1985.

6. R. REMMERT, Meromorphe funktionen in kompakten komplexen raümen, Math. Ann. 132 (1956), 277-283.

7. O. RIEMENSCHNEIDER, Characterizing Moišezon spaces by almost positive coherent analytic sheaves, Math. $Z .123$ (1971), 263-284.

8. O. RIEM ENSCHNEIDER, A generalization of Kodaira's embedding theorem, Math. Ann. 200 (1973), 99-102.

9. M. SCHNEIDER, Moishezon spaces and almost positive coherent sheaves, Math. Ann. 264 (1983), 517-524.

10. Y.T. Siv, A vanishing theorem for semipositive line bundles over non-Kähler manifolds, J. Diff. Geom. 19 (1984), 431-452.

11. Y.T. SIU, "Some recent results in complex manifold theory related to vanishing theorems for the semipositive case," Lecture Notes in Mathematics 1111, pp. 169-192.

12. A.J. SOMmese, Criteria for quasi-projectivity, Math. Ann. 217 (1975), $247-256$.

Departamento de Matemáticas

Centro de Investigación y de

Estudios Avanzados del I.P.N.

Apartado Postal 14-740

MEXICO 07000 D.F.

Rebut el 26 de Juliol de 1989 